\section{Spiral patterns in swirling flows}

Frédéric Moisy, Thomas Pasutto, Georges Gauthier, Philippe Gondret and Marc Rabaud

FAST, Bat. 502, 91405 Orsay Cedex, France

Cpiral galaxies, atmospheric or oceanic circulation, bathtub vor$\checkmark$ tices, or even stirring tea in a cup, are examples that illustrate the ubiquity of swirling flows at all scales in nature. They are not only fascinating, but also of great importance in a number of industrial or practical applications.

Earth rotation provides the most spectacular illustrations of rotating flows. At the end of the XIXth century, during the earlier polar expeditions, the Norwegian oceanographer Nansen noticed that the iceberg drift was not along the wind direction, as expected, but rather towards the right [1]. The Swedish physicist Walfrid Ekman, who saw the influence of the Coriolis force in this problem, gave an explanation for this phenomenon in 1905. For an observer in the Earth frame, a linear motion will appear as curved, with a deviation to the right in the Northern Hemisphere. Likewise, the upper layers of water, over a depth of about one hun dred meters, are dragged by the wind with a deviation towards the right [2]. The large oceanic motions originate from this phenomenon, and the same goes for the iceberg trajectories!

Let us consider a simple experiment, perhaps closer to our daily life. You have surely noticed that, when stirring tea, the tea leaves or other small solid particles heavier than water were collecting towards the centre of the bottom of the cup. Perhaps you would have expected the centrifugal force to expel them outwards! The friction at the bottom of the cup actually explains this seeming paradox. The centrifugal force, which varies as the square of the velocity, is weaker at the bottom, giving rise to a recirculation flow (see Figure 1). This inward recirculation is usually called the Bödewadt layer (1940), after the German fluid mechanician who described the motion of a rotating fluid over an infinite wall at rest. However, Albert Einstein was the first to give an explanation of this phenomenon in 1926 in the case of the teacup! [3] (It is said that, with this explanation, Einstein appeased Mrs. Schrödinger's curiosity, which her husband could not satisfy).

The region of fluid slowed down by the wall friction is called a boundary layer, and plays a key role in fluid mechanics. Its thickness, $\delta$, is given by the lengthscale where the imposed rotation $\Omega$ is diffused by viscosity in the intermediate fluid layers. In the ideal case of a fluid rotating over an infinite wall, the balance between centrifugal and viscous forces yields $\delta \approx(v / \Omega)^{1 / 2}$ (where $v$ is the kinematic viscosity of the fluid), which is a constant, independent of the radius $r$. Such a situation is said to be self-similar, i.e. the velocity profile remains unchanged when distances are rescaled. According to each situation, $\Omega$ may be the fluid velocity,
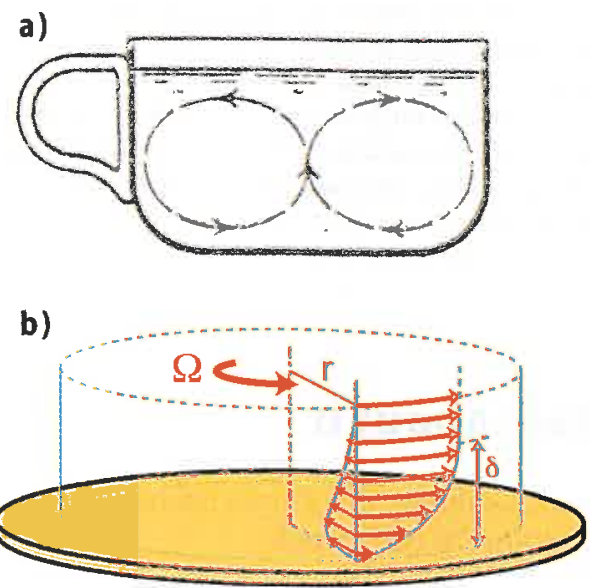

Aig. 1: (a) Original Figure by Einstein (1926), from his paper about the formation of meanders in the courses of rivers [3]. The rotation of the fluid is slowed down close to the bottom of the teacup, on a boundary layer of thickness $d$. (b) The centrifugal force in this layer is then much lower than in the rest of the fluid, giving rise to a recirculation flow which brings together the tea leaves in the centre of the cup.

the wall velocity or the relative velocity between the two. Within this self-similar description, since $\delta$ is the only lengthscale of the problem, all the physical phenomena are expected to take place on a scale of order $\delta$.

On the other hand, when the wall or the fluid extent is not infinite, other length scales, such as the teacup radius or the tea depth, may play a role too, and self-similar solutions are no longer of any help. Let us consider for simplicity the situation where the fluid is confined between two rotating disks-the upper one may be the free surface of the tea. In the general case, two boundary layers may be present, a centrifugal one over the faster disk and a centripetal one over the slower disk. Actually, the equations of motion without the self-similar hypothesis are so complex that no exact solution are known for this simple problem, even in the stationary regime. This problem gave rise to a famous controversy in the history of fluid mechanics: George Batchelor (1951) argued that two boundary layers, separated by a solid body rotation core, must take place in the fluid, whereas Keith Stewartson (1953)

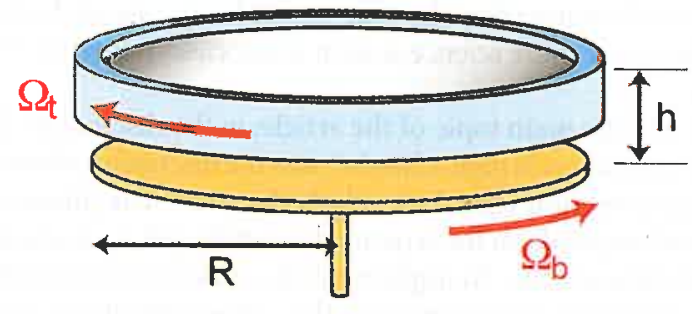

4 Fig. 2: Experimental set-up. The top disk is transparent, in order to allow visualisation from above. It rotates together with the cylindrical endwall (blue). The bottom disk (orange) is distant from the top disk by a few $\mathrm{mm}$ up to several $\mathrm{cm}$. In this picture, the bottom disk has been lowered for visibility. 
claimed that only one boundary layer should be present [4]. It has actually been shown, many years later, that a large variety of solutions may coexist in this flow, including the ones of Batchelor and Stewartson.

The stability of rotating flows is of considerable practical interest. Hard-disk drives are an important example: the instabilities of the thin air layer over the rapidly rotating platters induce vibrations of the read/write heads, that may damage the platters' surface [5]. The general problem of the stability of rotating flows is very complex, mainly because of two antagonistic effects: On the one hand, rotation tends to stabilise the flow, by inhibiting the perturbations about the rotation axis, eventually leading to a twodimensional state. On the other hand, the confinement generates differential rotation (basically because of the wall friction), leading to centrifugal forces imbalance and possible instabilities. In this context, there is no hope to obtain exact solutions, and only experiments or numerical simulations are able to shed light on the physical mechanisms responsible for the instabilities in rotating flows.

\section{A rotating disks experiment}

In order to study the instabilities of the flow between two rotating disks, the experimental set-up shown in Figure 2 has been built [6-8]. It consists of two coaxial disks, each of radius $R=14 \mathrm{~cm}$ and separated by a distance $h$, which can be varied between a few $\mathrm{mm}$ up to several $\mathrm{cm}$. The upper disk is the cover of a cylindrical rotating tank filled with a solution of water and glycerine, in which the lower disk can rotate independently. The upper disk is transparent, allowing us to visualise the flow from above. Small anisotropic flakes are seeding the working fluid, and their orientation with the velocity field leads to variations of the reflected light. For instance, the bright regions in the following pictures correspond to mainly horizontal flakes, whereas dark regions are associated with mainly vertical flakes.

Each disk rotates with its own angular velocity, $\Omega_{\mathrm{t}}$ and $\Omega_{\mathrm{b}}$. We call co-rotation the situation where both disks rotate in the same direction ( $\Omega_{\mathrm{t}}$ and $\Omega_{\mathrm{b}}$ are of the same sign); the instabilities of this flow are first described. The much richer patterns arising in the counter-rotating flow, when the two disks rotate in opposite direction, are analysed in a second part.

\section{Boundary layer instabilities}

Let us first consider the flow when only one disk, the upper one, rotates: this is the rotor-stator configuration $\left(\Omega_{t} \neq 0\right.$ and $\left.\Omega_{b}=0\right)$. When slowly increasing the disk velocity from 0 , nothing appears: the light reflected by the flakes remains homogeneous. The flow
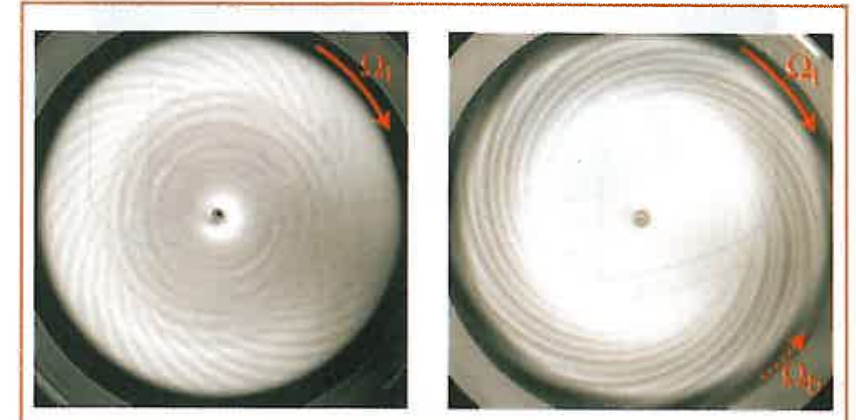

A Fig. 3: (a) Circles and positive spirals in the rotor-stator regime. These patterns result from boundary layer instabilities. (b) Negative spirals in the counter-rotation regime. This pattern originates from a shear layer instability.

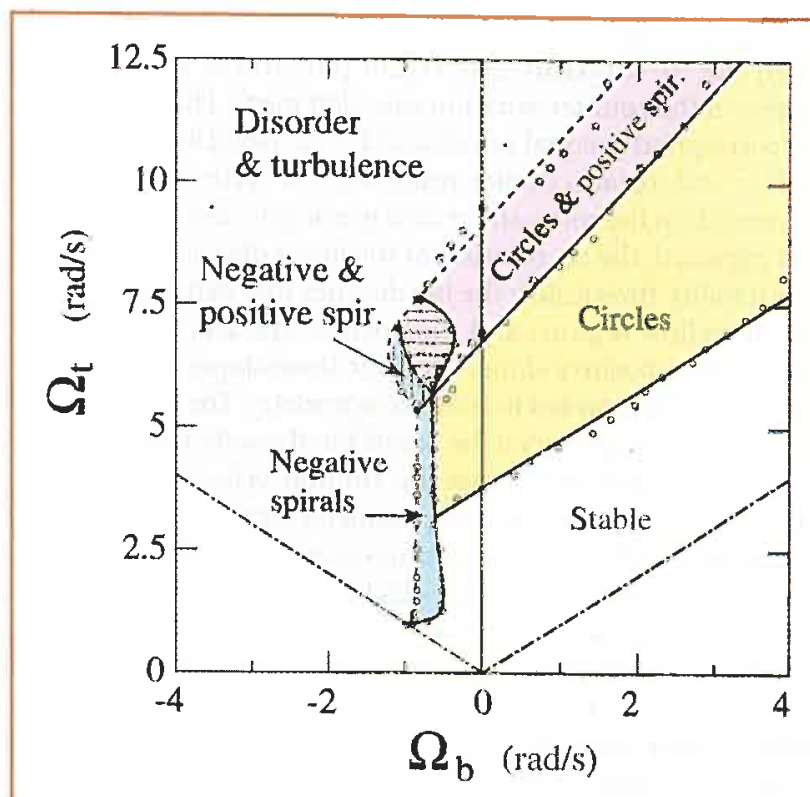

$\triangle$ Fig. 4: Regime diagram of the different instability patterns in the small gap case $(h=7 \mathrm{~mm})$. The right part $\left(\Omega_{\mathrm{b}}>0\right)$ corresponds to the co-rotation case, and the left part $\left(\Omega_{b}<0\right)$ to the counterrotation case [7].

may be seen as doubly symmetric: it is invariant with respect to any rotation (axisymmetric) as well as any time translation (stationary). Above a given disk velocity, a first instability pattern appears in the form of annular vortices, simply called circles, propagating towards the centre of the cell, as shown in Figure 3a. In this case, the temporal symmetry is broken, but the axisymmetry remains. If the angular velocity is further increased, another instability appears in the form of a spiral pattern. The axisymmetry of the flow is now broken too. This second pattern received the name of positive spirals, because they roll up to the centre in the direction of the rotating disk.

A careful inspection of the Figure 3 a allows us to understand the nature of the instability that gives rise to these patterns. One can see the spiral arms do not extend over the whole flow, but rather stop at a well-defined radial location, where the boundary layers of each disk merge. In other words, for $r>r_{0}$, where the positive spirals can be seen, the boundary layers are separated, whereas for $r<r_{0}$ the viscous effects dominate the flow over the whole gap and no boundary layer can be defined. This observation suggests that positive spirals only exist within the boundary layers, and that they are the result of boundary layer instability. Additional observations, by means of visualisations in the vertical plane normal to the disks, confirm this assumption.

What happens now if the lower disk rotates too? From the frame rotating with the lower disk, this situation is similar to the one where only the upper disk rotates with a relative angular velocity $\Delta \Omega=\Omega_{\mathrm{t}}-\Omega_{\mathrm{b}}$. As a consequence, the instability threshold should just get shifted upwards, of a quantity $\Omega_{b}$. Unfortunately, this picture is rather naive: the dynamics in a rotating frame is very different from that in the laboratory frame. In order to take into account the non-Galilean nature of the rotating frame, one should consider the effect of the Coriolis force on the instabilities.

We show in Figure 4 a diagram that summarizes our observations when both disks are rotating. The vertical and horizontal axes correspond to the angular velocities of the bottom and top 
disks. By convention, $\Omega_{\mathrm{t}}$ is always positive, while $\Omega_{\mathrm{b}}$ may be positive in the co-rotation case (right part of the diagram) or negative in the counter-rotation case (left part). The two dashed lines correspond to equal velocities: $\Omega_{\mathrm{t}}=\Omega_{\mathrm{b}}$ (solid body rotation) and $\Omega_{\mathrm{t}}=-\Omega_{\mathrm{b}}$ (exact counter-rotation). The vertical line, $\Omega_{\mathrm{b}}=0$, corresponds to the rotor-stator case previously described.

As expected, the co-rotation of the lower disk shifts upwards the instability thresholds: the borderlines that delimit the circle pattern (yellow region) and the positive spirals pattern (pink region) have a positive slope. However, these slopes are different, which can be interpreted in terms of symmetry. The borderline of the circle pattern appears to be parallel to the solid body rotation line, $\Omega_{\mathrm{t}}=\Omega_{\mathrm{b}}$, indicating that the angular velocity difference $\Delta \Omega=\Omega_{\mathrm{t}}-\Omega_{\mathrm{b}}$ is the only control parameter for this instability, and no influence of the global rotation occurs. In other words, the instability responsible for the circle pattern, which does not break the axisymmetry, is not affected by the additional rotation of the frame, i.e., by the Coriolis force. By contrast, the borderline for the positive spirals, which are responsible for the axisymmetry breaking, has a larger slope than the solid body rotation line: in this case, the relative angular velocity $\Delta \Omega=\Omega_{\mathrm{t}}-\Omega_{\mathrm{b}}$ is not the only control parameter, and an extra velocity of the upper disk is needed for the positive spirals to arise. The global rotation has now the expected stabilising effect mentioned in the introduction.

\section{Shear flow instability}

So far we have restricted our attention to the co-rotative $\left(\Omega_{\mathrm{b}}>0\right)$ and weakly counter-rotating (about $\Omega_{\mathrm{b}}>-0,5 \mathrm{rad} / \mathrm{s}$ for this gap) regimes. The observed phenomena are rather different if we now focus on a more intense counter-rotating regime, where a new instability pattern arises, as shown in Figure 3b. Here again we observe a spiral pattern, but it is by far very different from the boundary layer instability patterns described up to now. First, the spiral arms roll up the centre in the direction opposite to the faster disk: for this reason we call theñ negative spirals (blüe region in the diagram of Figure 4).

Perhaps the most striking characteristic of the negative spirals is their very large growth time: when the onset is carefully approached from below, about 10 to 20 minutes are required for the negative spirals to Perhaps the most arise. Such very slow dynamics strongly contrasts with the

\section{striking characteristic}

of the negative spirals circles and positive spirals, which appear almost instantaneously when their threshold is reached. For this reason, a precise determina-

is their very large tion of the negative spirals growth time threshold is a rather delicate work, that needs a very stable and controlled apparatus and... a lot a patience! Slightly further from the threshold, this growth time takes more reasonable values, of the order of one minute or a few seconds. Actually, it can be shown that this growth time diverges as one approaches the onset, a usual property for critical systems near a bifurcation point.

What happens now if the gap between the disks is changed? We can see that the morphology of the negative spirals strongly differs, from $h=7 \mathrm{~mm}$ (Figure $3 \mathrm{~b}$ ) to $h=20 \mathrm{~mm}$ (Figure 5a). For this new gap, the instability gives rise to a more complex structure near the centre of the cell, in the form of a circular chain of vor-
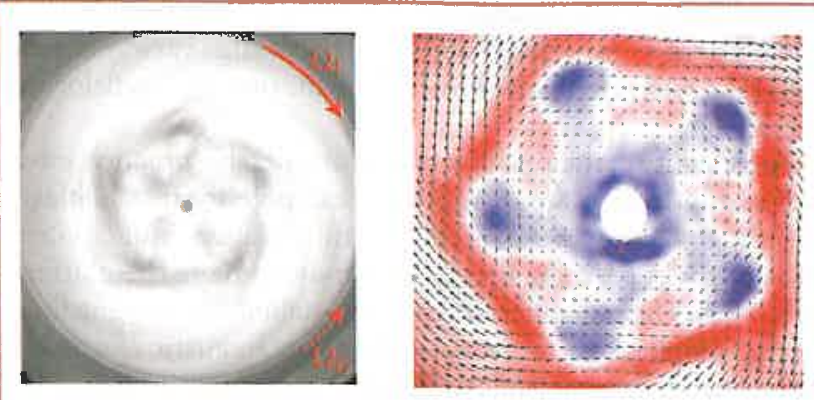

$\triangle$ Fig. 5: (a) Example of a 5-armed negative spiral pattern, observed for a large gap thickness $(h=20 \mathrm{~mm})$. This picture has to be compared to the equivalent pattern for a small gap, in Figure $3 \mathrm{~b}$. (b) Corresponding velocity field, as measured from Particle Image Velocimetry (PIV). Colors are coding the levels of vertical vorticity, i.e., the $2 \mathrm{D}$ local rotation rate of fluid particles [8].

tices surrounded by spiral arms. Moreover, the number of spiral arms is smaller in the large gap case: from 11 arms for the small gap, down to 5 arms in the large gap case (values down to 2 arms can be found for even higher values of $h$ ). This variation can be easily understood, if we imagine the spiral arms as vortex tubes, whose diameter is of order of the gap thickness $h$.

More insight into the physical mechanism responsible for the formation of this pattern may be obtained from the velocity field of the bifurcated flow. In the case of the large gap, this velocity field can be obtained by means of Particle Image Velocimetry (PIV). This non-invasive technique consists in measuring the distance swept by small particles seeding the flow between two successive images. The particles are illuminated by a pulsed laser sheet synchronised with a high-resolution video camera. In Figure 5b, obtained for the same angular velocities as in Figure $5 \mathrm{a}$, we can see the circular chain of 5 vortices surrounded by the negative spiral arms, similar to the pattern visualised using the flakes [8]. The colours encode the levels of vorticity, i.e., of local rotation rate of the fluid particles, from blue (counter-clockwise) to red (clockwise).

An important feature of this velocity field is the presence of an intense shear layer (in red), where strong vorticity is concentrated.

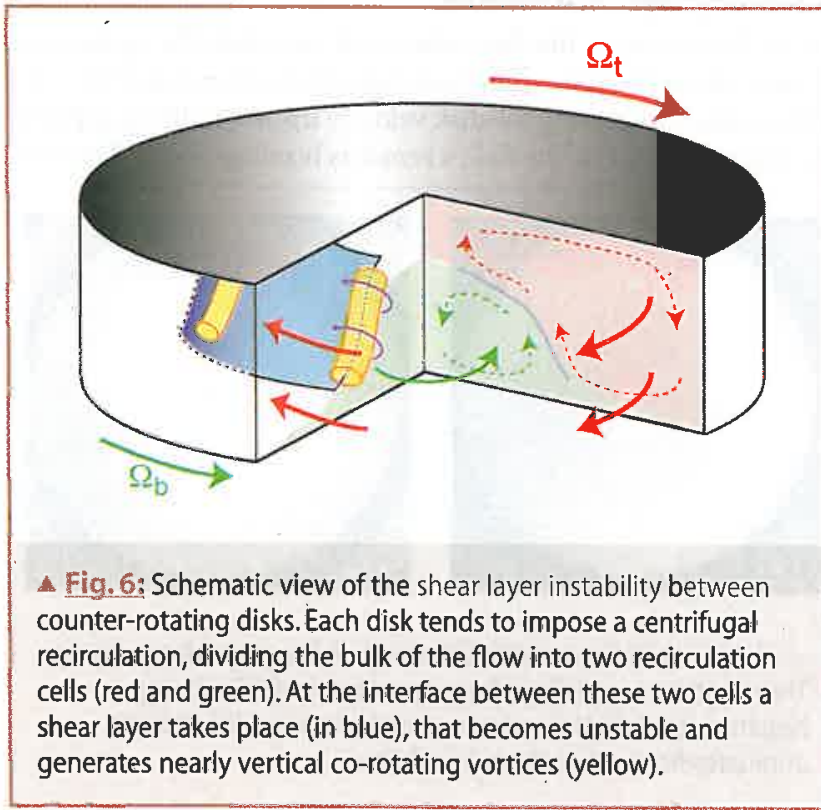




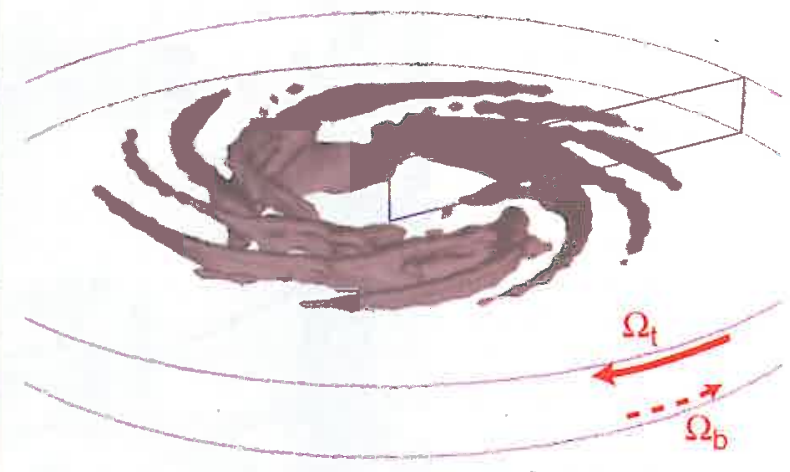

A Fig. 6: Numerical simulation of the flow between counterrotating disks. Negative spirals with 9 spiral arms can be seen. Data from O. Daube and P. Le Quéré [10].

This thin layer separates the outer part, rotating with the faster (upper) disk, from the inner part, where much lower velocities are found. The origin of this shear layer can be understood from the Figure 6 . In the counter-rotating regime, each disk tends to impose its rotation to the fluid (full arrows), associated with a centrifugal flow (dashed arrows). The centrifugal flow induced by the faster disk, in red, recirculates towards the centre of the slower disk due to the lateral end wall. This inward recirculation flow meets the outward radial flow induced by the slower (bottom) disk, in green, leading to the formation of two recirculation cells. At the interface between these two cells a strong shear layer takes place. Such layer is prone to an instability, which leads to an azimuthal modulation and to the roll-up into individual co-rotating vortices [9]. This instability mechanism was first introduced in the simpler case of a linear shear layer by Lord Kelvin and Hermann ... he avy century, and was aiming to explain the wave formation due to the wind stress on the sea surface [2].

Our flow between rotating disks, although very simple, presents two classes of instability patterns, associated with very different physical mechanisms: boundary layer instabilities (circles and positive spirals), which have been studied for a long time in similar flow geometries, and shear layer instability (negative spirals), which have been first observed in our particular set-up between counter-rotating disks. The complexity of the observed phenomena is striking compared to the apparent simplicity of the flow geometry. This is a generic situation for systems governed by nonlinear equations, among which the hydrodynamics systems play a central role. The basic solutions, usually simple because associated with a high degree of symmetry, are replaced by much richer patterns, that may coexist and interact together (see the dashed regions in Figure 4). The flow between two coaxial cylinders, of practical importance in rheology, is another example of very simple flow with a large variety of instability patterns and transitions towards turbulence.
The new class of instability revealed in our experiment has motivated a numerical study of the flow between counter-rotating disks. Such simulations are very expensive in terms of computational time: because of the very large growth times, the full 3D Navier-Stokes equations have to be simulated over a very long time. This work has been carried out by Olivier Daube, of laboratory CEMIF (Evry, France), and Patrick Le Quéré, of laboratory LIMSI (Orsay, France). Figure 7 is a visualization of the flow between counter-rotating disks separated by the distance $h=20 \mathrm{~mm}$, where the surfaces of iso-vertical velocity are shown [10]. This quantity traces the rolling up of the streamlines in the radial and azimuthal directions, and clearly exhibits a spiral pattern in excellent agreement with the ones observed experimentally.

Such heavy computations are now feasible thanks to new generation of supercomputers, and opens new and exciting perspectives in the understanding of complex flow phenomena. Among the situations of considerable practical interest are the turbomachines used in power plants or aeronautics engineering. This latter application involves huge rotations rates (more than 10 $000 \mathrm{rpm}$ ), and accurate modelling of the turbulent phenomena present at small scales are clearly needed for such numerical simulations. In this context, the excellent recent agreements between experiments and numerical simulations are encouraging for the understanding and modelling of turbulence under strong rotation.

This article is based on an original version published in Bulletin de la SFP (French Physical Society), 135, p.4, July 2002.

\section{References}

[1] http://www.nrsc.no/nansen/fritjof_nansen.html

[2] E. Guyon, J.P. Hulin, L. Petit and C. D. Mitescu, Physical hydrodynamics (Oxford University Press, 2001).

[3] A. Einstein, Die Naturwissenschaften 26, 223 (1926). A translation can be found in Ideas and Opinions (Bonanza Books, New York, 1954), pp. 249-253.

[4] G.K. Batchelor, Q.J. Mech. Appl. Maths 4, 29 (1951). K. Stewartson, Proc. Camb. Phil. Soc. 49, 333 (1953).

[5] J.A.C. Humphrey, C.J. Chang, H. Li and C.A. Schuler, Adv. Inf. Storage Syst. 1, 79 (1991).

[6] G. Gauthier, P. Gondret and M. Rabaud, J. Fluid Mech. 386, 105 (1999).

[7] G. Gauthier, P. Gondret, F. Moisy and M. Rabaud, J. Fluid Mech. 473, 1 (2002). See also Phys. Fluids 14, S7 (2002), and the Gallery of Fluid Motion http://ojps.aip.org/phf/gallery/index1.jsp

[8] F. Moisy, T. Pasutto and M. Rabaud, Nonlinear Processes in Geophysics 10, 1 (2003).

[9] J.M. Lopez, J.E. Hart, F. Marques, S. Kittelman and J. Shen, J.Fluid Mech. 462, 383 (2002)

[10] O. Daube, P. Le Queré, F. Moisy and M. Rabaud, Proceeding 2nd International Conference on Computational Fluid Dynamics (2002). 